\title{
El desarrollo de una conducta lectora a través del aprendizaje significativo
}

\section{Ma. Trinidad Román*}

\section{RESUMEN}

En este ar tícu lo se abor da el pro ble ma del de sa rrollo del gus to por leer des de el pun to de vis ta de la Psi colo gía Hu ma nis ta, es pe cíficamente de la corriente educativa llamada Educación Centrada en la Persona, la cual aprovecha los aportes de la psicología mencionada para aplicarlos a la educación.

\section{SUMMARY}

This pa per approa ches the pro blem of de ve lo ping the peo ple's plea su re of rea ding from the point of view of the Hu ma nis tic Psycho logy, it ta kes as re fe ren ce the cu rrent ca lled Per son Cen te red Edu cac tion, which takes ad van te ge of the con tri bu tions of this psychology in order to apply it to education.

Todavía — señala la UNESCO ${ }^{1}$ — no existe ningún país que se sien ta sa tis fe chocon la can ti dad y/o ca li dad de lec tu ra de la mayoría de sus habitantes.

En Mé xi co, en una in ves ti ga ción rea li za da en 1985 por la Dirección Ge ne ral de Bi blio te cas de la Se cre ta ría de Edu ca ción Pública $^{2}$, se en con tró que fue ra de los ci clos es co la res, la mayoría de las personas sí leían, pero sus lecturas selimitabana ma te rial que se pue de ca li fi car como cha ta rra (v.g. fo to no velas).

A su vez, se considera que un alto porcentaje de estudiantes leen solamente lo estrictamente necesario para cumplir con sus obligaciones escolares, pero no les gusta leer; lo encuentran aburrido.

Para promover el comportamiento lector se han organizado diversos programas de fomento del libro tales como ferias, exposiciones, campañas, festivales... en fin, diferentes tipos de actividades promocionales ${ }^{3}$. Sin embargo, mi apreciación personal es que estos eventos incrementan la lectura principal men te en los lec to res cau ti vos, pero no tie nen mu cho efecto so bre las per so nas re nuen tes a leer, o a leer cier ta ca li dad de ma te rial, que no sean fo to no ve las o his to rie tas; y a quie nes se considera que son la mayoría.

No bas ta al fa be ti zar, para lo grar que las per so nas lean, es necesario que éstas quieran hacerlo; porque disfrutan de ello. Además, este gusto por leer no es algo que se da espontáneo, sino que implica, además de la habilidad de leer y la posibilidad de tener acceso a los libros, el desarrollo de una actitud positiva hacia la lec tu ra, que ge ne ral men te se for ma en la niñez.

Este gusto por leer, no es algo innato, no nacemos lectores o no lectores, nos hacemos una cosa u otra a través de nuestras experiencias de lectura.

Si se observa, el niño pe que ño que to da vía no ha asis ti do a la escuela, tiene una actividad incesante; su "curiosidad es ilimi ta da, quie re ave ri guar, ha cer, for mar, crear. Ab sor be in formación a través de sus ojos, oídos, nariz, boca y dedos. Está asimilando conocimientos, percibiendo pautas, adquiriendo un idioma y perfeccionando destrezas. Aprende, aprende y aprende, a un ritmo que probablemente nunca igualará."4 .

Luego va a la escuela. Las posibilidades de expansión de sus ca pa ci da des son casi in fi ni tas, pero por lo ge ne ral se en cuentra con mé to dos, ac ti tu des e in for ma ción que so fo can sus tenden cias al de sa rro llo. El ma te rial que se le pre sen ta al niño en clase, es abstruso y carente de significado, de manera que la educación se convierte en un esfuerzo de adquirir información poco re le van te para su vida e in te re ses. En tal apren di zaje, sólo in ter vie ne la men te, pero sin par ti ci pa ción de las emociones, ni de las significaciones personales, de suerte que no reviste importancia para todas las par tes que in te gran su persona.

"Hay prue bas - afir ma Ro gers- de que a me di da que los niños pasan por el sis te ma de en se ñan za, se vuel ven me nos inquisitivos y menos curiosos"5 (condición indispensable para el de sa rro llo del gus to por leer), y ésta, es una de las acu sa ciones más graves que se le puede hacer al sistema educativo.

Además, el bajo índice de buenos lectores es otra prueba del pocoéxi to que ha te ni do la es cue la, con re la ción al de sa rrollo de una conducta lectora, entre quienes enseña a leer.

Aho ra bien, por lo vis to an te rior men te, se pro po ne abor darel problema de la formación de lectores desde el punto de vista

* Investigadora del Centro Universitario de Investigaciones Bibliotecológicas de la UNAM 
delapsicologíahumanista, es pecífica men telacorrienteeducativa llamada Educación Centrada en la Persona.

Esta corrien te, re la tiva men te jo ven, surge de la técnicapsicoterapéutica propuesta por Carl Rogers, quien a través de sus numerosas investigaciones en la psicoterapia comprobó que lo que real men te ayu da ba y pro pi cia ba cam bios en una per sona, no era tanto la técnica empleada por el terapeuta, sino la capacidad de éste para establecer un tipo de relación basada en ciertas actitudes que facilitaban el crecimiento de las personas.

Así, que si este tipo de relación producía resultados sorprendentes en la terapia, ¿por qué no aplicarla a la educación?

Es tas ac ti tu des no son técnicas o des tre zasin natas delfa cilitador del aprendizaje ${ }^{6}$, sino que las tiene que desarrollar. Tampoco son reglas metodológicas exteriores para estructurar la relación, sino que son condiciones actitudinales, que al ser experimentadas por el facilitador implican un desarrollo diná mi co en la re la ción; lo que per mi te crear una si tua ción en la cual, des de el prin ci pio, se des car ta la de pen den cia, se pro picia que el es tu dian te sea él mis mo, se pro mue ve su liber tad y responsabilidad, y le permite desarrollar la confianza en él mis mo. En otras pa la bras, fa vo re ce que el es tu dian te des pliegue sus potencialidades.

Estas condiciones de actitud para establecer la relación son:

\section{Aceptación incondicionada al estudiante}

Una de las actitudes de los que tienen éxito en la facilitación del aprendizaje es apreciar al alumno, sus sentimientos, opinio nes y toda su per so na. Es de cir, la acep ta ción del otro in dividuo como una persona independiente, con derechos propios. Así como un respeto absoluto por el estudiante a quien se con tem pla como una per so na dig na, va lio sa, con de re cho a ser un individuo libre. De esta manera, "el alumno puede ser él mis mo, sin te mor a ser re chaza do, cas ti ga do, o no valo ra do por su maes tro, quien con fía en él y en su po ten cial hu ma no." 7

La acep tación in con di cional re quiere, por su pues to, de un clima de per mi si vi dad y li ber tad. Lo que no im plica la au sen cia delímites. Tampocoelaceptarincondicionalmente, significa la apro ba ción de una con duc ta des truc ti va. Se pue de acep tar a la per sona, peroes tar en de sa cuer do y de sa probar la con ducta que le hace daño a sí misma, a sus semejantes, o a ambos.

\section{La comprensión empática}

Cuando el profesor tiene la capacidad de comprender las reacciones del estudiante desde el marco de referencia de éste, podrá facilitar un aprendizaje significativo.

Esta actitud de ponerse en el lugar del otro, de ver el mundo des de el pun to de vis ta del es tu dian te, sin cri ti car lo, juz garlo, ni eti que tar lo, es casi ine xis ten te en el aula tra di cio nal, "don- de es posible escuchar — señala Rogers - miles de interacciones, sin encontrar un solo ejemplo de comunicaciónclara, sensible y empática. Pero cuan do esto ocu rre, tie ne un efec to de to tal li be ra ción... Si todo maes tro se pro pu sie ra la ta rea de es for zar se para brin dar cada día una res pues tanoevalua tiva, sino com pren si va y em pática a la vi ven cia ma ni fes ta da o verbalizada de un estudiante, creo que descubriría todas las potencialidades de este tipo de comunicación." 8

\section{La congruencia}

El apren di zaje se gún Rogers, pa re ce serfacilita do si el maes tro es con gruen te. Es de cir, si el maes tro se pre sen ta tal como es, sin caretas ni disfraces, posee un amplio conocimiento y aceptación de sus sentimientos y de sus actitudes, y es capaz de entablar una relación interpersonal igualitaria con sus alumnos; entonces, el clima para el aprendizaje se engrandece.

Así, que a través de la creación de esta atmósfera de aceptación incondicional, comprensión empática y congruencia; y por otra parte, dentro de un marco de libertad se encausa el aprendizaje, a partir de lo que es im por tan te en la vida del estudiante, y tratando de propiciar experiencias vivenciales e integrales, se facilita el aprendizaje significativo y además sucede, que se vuelve autogestivo.

Este tipo de apren di za je sig nifica ti vo es el que sus ten ta la corriente educativa centrada en la persona.

Este aprendizaje, se podría definir como "el aprendizaje que tie ne sen ti do en la vida de quien apren de, es de cir, que le es interesante, motivante, incluso necesario para su desarrollo y rea li za ción per so nal. Este tipo de apren di za je se asi mi la al organismo, al igual que lo hace el alimento que se ingiere para satisfacer una ne ce sidad, se di gie re por te ner ele men tos nu tritivos y se aprovecha. Por lo tanto, al hablar del aprendizaje significativo nos referi mos a un apren di zaje que es asi mila do con sentido." Además este tipo de aprendizaje siempre implica un contenido afectivo.

La lec tu ra es un apren di za je que si ha de ser efec ti vo, im pli ca no sólo la habilidad de descifrar signos, sino la vivencia de una experiencia integral, que sea significativa en la vida de quien estále yen do. Sólo en ton ces se po drá de sa rro llaruna actitud positiva hacia la lectura, y por lo mismo, una conducta lectora deseable.

"El niño que con dificultad aprende a leer, un día se interesa por un re la to es cri to, y se da cuen ta de que las pa la bras tie nen un poder mágico que lo llevan a mundos hasta ese momento desconocido. Entonces, y sólo entonces, ha aprendido 'realmente' a leer." 10

Ahorabien, las ca rac te rís ti cas que ge ne ra la lec tu rasig nificativa, favorecida por el clima descrito, serían:

1. Lo leído se asimila al plano del ser, no del tener. Quien lee 
algo de esta ma ne ra, no sólo tie ne más en su ha ber, sino que es más como persona.

2. Lo que se lee significativamente, se integra con los conocimien tos y las ex pe rien cias an te rio res. Lo que lle va a pro vo car el sentido crítico del estudiante.

3. Lo leído significativamente, se asimila y queda como un recurso que puede ser utilizado en cualquier momento.

4. La lectura significativa es autoiniciada, la persona elige el tema que verdaderamen te le in te re se, y se fo men ta sus iniciati vas y su ge ren cias, lo que re dun da en un in cre men to de lec turas sig ni fica ti vas. De esta ma ne ra, tam bién se li be ra la cu riosidad innata de la persona y se estimula su deseo de leer.

5. La lectura significativa es una comunicación con nosotros mismos. El lector recrea y crea nuevamente la obra que lee.

6. La per so na que lee sig nifica ti va men te estáca pa ci ta da para reflexionar y apreciar el valor de esa lectura en su vida, y sólo ella, puede autoevaluar la trascendencia de esa lectura.
En resumen, "Rogers propone la educación centrada en la per so na, con base en la hi pó te sis hu ma nis ta de que el ser humano es digno de confianza y respeto; y tiene desde su nacimiento una capacidad de autodirección, que le permite la toma de decisiones y la elección de sus propios valores.

"Este sistema edu ca ti vo es un pro ce so di ná mi co, en el cual el estudiante participa activamente en el proceso de su propio aprendizaje y desarrollo integral. El estudiante que participa en este proceso educativo-señala Ana Ma. González- desarrolla la creati vidad, se des cu bre como ser libre, y com prende su propio ambiente al comprometerse con éste." 11

Por lo tan to, abor darel de sa rrollo de la con duc ta lec to ra des de la corrien te del apren di zaje sig nifica ti vo, pa re ce ser uncamino pro me te dor, que ten drá re per cu sio nes no sólo en la for mación de lectores sino también de personas más maduras.

\section{CITAS}

1 UNESCO. "Con gre so mun dial del li bro" Lon dres, 7-11 de ju nio de 1982.Recomendaciones Generales 1982. p. 5 (COM-82/CONF. 401/COL 9).

2 Bibliotecas públicas y conducta lectora: Investigaciones 2. México, Consejo Nacional para la Cultura y las Artes. Dirección General de Bibliotecas, 1989. 150 p.

3 Para mayor información sobre el tema, puede consultarse a:

STAIGER, Ralph. Caminos que llevan a la lectura. Paris, UNESCO, 1979.

STAIGER, Ralph y Claudia Casey. Planificación y organización de campañas de lectura; guía para países en desarrollo. Paris, UNESCO, 1983.

4 ROGERS, Carl R. Libertad y creatividad en la educación en la década de los ochenta. México, Paidos, 1986. p. 346.

$5 \quad$ Idem. p. 167.

6 En este ar tícu lo se en tien de por fa ci li ta dor al pa dre, maes tro o bi blio te ca rio que de sea pro pi ciarel aprendizaje en la otra persona.

7 GONZALEZ GAR ZA, Ana Ma ría.El enfo que cen trado en la per so na; aplica cio nes a la edu cación. México, Trillas, 1987. p. 90.

$8 \quad$ ROGERS, Carl R. op. cit. p. 150.

9 GONZA LEZ GAR ZA, Ana Ma ría.Elaprendizajesignificativo. Po nen cia pre sen ta da en el Co legio Peninsular Rogers Hall, en Mérida Yucatán en 1988. p. 1 (no publicada).

10 ROGERS, Carl R. Libertad y creatividad en la educación. 1a. ed. México, Paidos, 1968. p. 14.

11 GONZALEZ GARZA, Ana María. op. cit. p. 14. 


\section{BIBLIOGRAFIA CONSULTADA}

1 Bibliotecas públicas y conducta lectora: Investigaciones 2. México, Consejo Nacional para la Cultura y las Artes. Dirección General de Bibliotecas, 1989. 150 p.

2 EGUINOA, Ana Es ter.Didácticauniversitariadelalectura. México, UniversidadVeracruzana, 1987. $108 \mathrm{p}$.

3 GONZA LEZ GARZA, Ana María.Elaprendizajesignificativo. Po nen cia pre sen ta da en elCo legio Peninsular Rogers Hall, en Mérida Yucatán en 1988. 11 p (no publicada).

4 GONZALEZ GAR ZA, Ana Ma ría.El enfo que cen trado en la per so na; apli ca cio nes a la edu cación. México, Trillas, 1987. 174 p.

5 GONZALEZ GARZA, Ana María. "Los modos del cerebro y el aprendizaje significativo." En: DIDAC, bole tíndel Centrode Didác ticadela Univer sidadIbe ro ame ricana; Pri ma ve ra 87; Art. 47 .

6 JOLIBERT, Josette y Robert Gloton. El poder de leer. Barcelona, Ed. Edisa, 1985. 336 p.

7 ROGERS, Carl R. “EEl aprendizaje pue de in cluir tan to ideas como sen ti mien tos?” En: LA FARGA CO RO NA, Juan y José Gó mez del Cam po.Desarrollodelpotencialhumano. 2 ed. México, Trillas, 1989. 3 vol.

8 ROGERS, Carl R. Libertad y creatividad en la educación en la década de los ochenta. México, Paidos, 1986. 364 p.

9 UNESCO. "Con gre so mun dial del li bro" Lon dres, 7-11 de ju nio de 1982.Recomendaciones Generales 1982. 11 p. (COM-82/CONF. 401/COL 9)

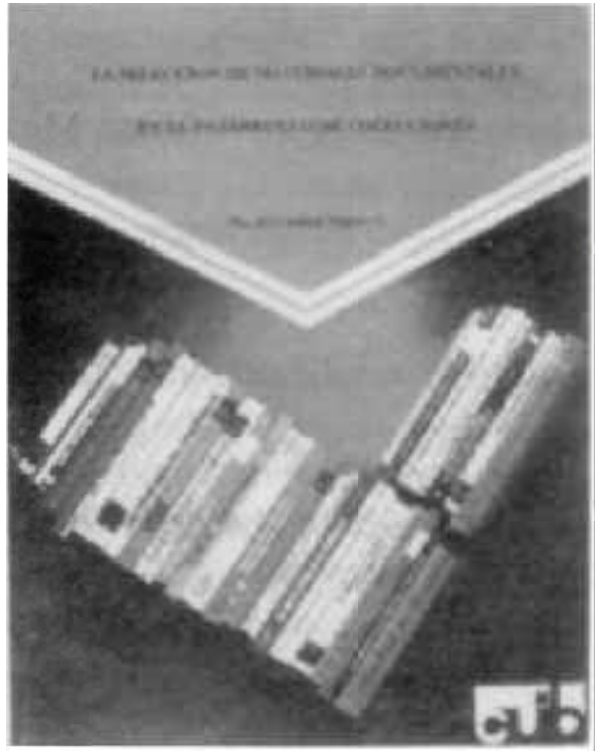

La selección de materiales documentales en el desarrollodecolecciones. Ne gre te, Ma. del Carmen $102 \mathrm{p}$.

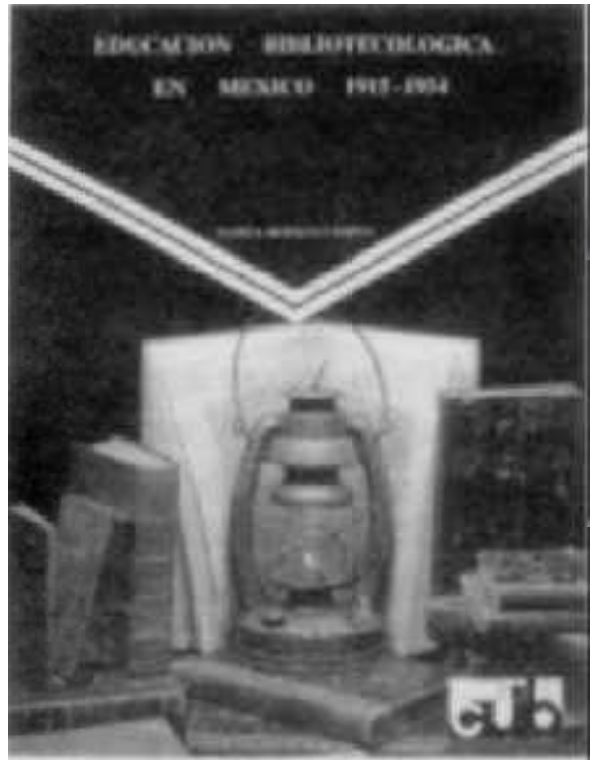

Educación bibliotecológica en México 1915-1954. Morales, Estela $110 \mathrm{p}$. 